distress alone were more likely at the higher end of the socioeconomic distribution

\section{OP50 ALCOHOL INTAKE, DRINKING BEHAVIOUR AND DRINKING PATTERNS AS PREDICTORS OF EMPLOYMENT STATUS IN WORKING-AGE MEN IN IZHEVSK, RUSSIA}

doi:10.1136/jech-2012-201753.050

S A Cook, D A Leon. Faculty of Epidemiology and Population Health, London School of Hygiene \& Tropical Medicine, London, UK

Background Hazardous alcohol consumption and unemployment are both associated with high mortality in Russia. Unemployment adversely affects mental and physical health. The relationship between alcohol and employment is complex as alcohol consumption may lead to unemployment but unemployment may also increase hazardous consumption. Very few longitudinal studies have investigated the effects of alcohol use on employment status. The objectives of this study were to investigate alcohol intake, drinking behaviour and drinking patterns as predictors of employment status in a longitudinal study of working-age men in Izhevsk, Russia.

Methods Participants were 1217 men aged 25-54 resident in Izhevsk, Russia in regular paid employment at baseline (2003-6) who were followed up and re-interviewed (2008-9). Alcohol use was measured by total volume of ethanol consumed from beverage alcohol, drinking behaviour (abstainers, beverage alcohol drinkers with no problem drinking behaviour, beverage alcohol drinkers with problem drinking behaviour and non-beverage alcohol drinkers (e.g. eau de cologne)), and three aspects of drinking pattern (drinking spirits without food, drinking alone and drinking before noon). Problem drinking was defined as twice weekly or more frequency of hangover, excessive drunkenness, sleeping in clothes because of drunkenness or one or more episodes of zapoi in the past year (defined as continuous drunkenness of two or more days during which participants were withdrawn from normal life). Logistic regression was used to investigate whether alcohol use at baseline predicted employment status at follow up after adjusting for sociodemographic variables.

Results Total volume of ethanol from beverage alcohol at baseline did not predict whether men were still in regular paid employment at follow-up ( $\mathrm{P}$ value for linear trend=0.21). Problem drinkers (adjusted odds ratio 2.82 95\% CI 1.23, 6.46) and non-beverage alcohol drinkers (adjusted odds ratio 2.42 (95\% CI 1.18, 4.93) were more likely not to be in regular employment at follow-up than non-problem beverage alcohol drinkers. Among drinkers drinking spirits without food (adjusted odds ratio 2.13 95\% CI 1.28, 3.54), drinking alone (adjusted odds ratio $1.6595 \%$ CI 1.09, 2.50) and drinking before noon (adjusted odds ratio 2.49 95\% CI 1.65, 3.78) all predicted employment status at follow-up.

Conclusion Drinking behaviour and drinking patterns predicted future employment status in a sample of working-age men in Izhevsk Russia. However the volume of alcohol consumed per year did not, suggesting how alcohol is consumed and whether it leads to problem behaviour is more important than the overall amount consumed when considering the impact of alcohol on employment.

\section{OP51 MATERNAL EMPLOYMENT AND SOCIO-EMOTIONAL BEHAVIOUR AT AGE 7: FINDINGS FROM THE UK MILLENNIUM COHORT STUDY}

doi:10.1136/jech-2012-201753.051

SC Hope, A Pearce, C Law. MRC Centre of Epidemiology for Child Health, UCL Institute of Child Health, London, UK

Background Maternal employment in the UK has increased significantly in recent years, but evidence of its relationship with child socio-emotional behaviour is mixed. Given the policy importance of both family employment and children's wellbeing, this relationship needs to be better understood. This study seeks to investigate whether cumulative exposure to maternal employment is associated with socio-emotional behaviour at age 7 .

Methods The study comprises a longitudinal analysis of the United Kingdom Millennium Cohort Study (MCS), based on 10,723 singleton children who participated in all four sweeps of the study (at ages 9 months, 3, 5 and 7 years). Socio-emotional behaviour was measured using total Strengths and Difficulties Questionnaire (SDO) scores, dichotomised using an established cut-off for borderline / abnormal behaviour. Risk ratios (RR) were estimated using Poisson regression for borderline / abnormal SDQ scores at age 7 according to cumulative maternal employment over four sweeps of the MCS (at 9 months, 3, 5 and 7 years), unadjusted (uRR) and adjusted (aRR) for confounders (age at first live birth, ethnicity, family size, lone parenthood, and maternal psychological distress, all recorded at 9 months). In order to disentangle whether there was a differential impact of earlier and current employment, additional analyses estimated the risk associated with employment up to age 5 years, adjusting for employment at age 7 .

Results The risk of borderline/abnormal scores decreased linearly by number of MCS sweeps that mothers were in employment; after adjustment for confounders the gradient attenuated but remained significant. Children growing up in households where mothers were employed in all MCS sweeps were significantly less likely to display borderline / abnormal behaviour at age 7 (compared to never in paid employment): $\mathrm{uRR}=0.37$ (0.32-0.44); $\mathrm{aRR}=0.68$ (0.56-0.81). Analyses differentiating early employment (between ages 9 months and 5 years) and current employment (at age 7) suggest that the cumulative impact of prior non-employment may be ameliorated once a mother gains employment. After adjusting for current employment and confounders, there was no increased risk of borderline / abnormal socio-emotional behaviour associated with non-employment in all earlier sweeps up to age 5 compared to being in paid employment continuously (baseline): $\mathrm{aRR}=1.01(0.82-1.23)$.

Conclusion This longitudinal analysis supports an association between maternal employment and child socio-emotional behaviour. Possible explanations and implications will be discussed.

\section{OP52 LONG-TERM EXPOSURE TO INCOME INEQUALITY: IMPLICATIONS FOR PHYSICAL AND MENTAL HEALTH AT OLDER AGES}

doi:10.1136/jech-2012-201753.052

R de Vries, D Blane, G Netuveli. Primary Care and Public Health, Imperial College London, London, UK

Background The 'inequality hypothesis' proposes that higher levels of societal income inequality have a detrimental effect on both physical and mental health. Previous studies have provided only mixed support for this hypothesis, particularly among older people. However, by using only contemporary income inequality estimates, or estimates from a single lag period, the majority of previous studies have not accounted for people's continued exposure to income inequality over the long-term. In this study, we addressed this problem by examining the association between older people's experience of inequality over an extended period and their subsequent health.

Methods The data for this project were taken from three comparable nationally representative surveys (covering 16 countries) of the health and circumstances of older people; the English Longitudinal Study of Ageing, the Survey of Health and Retirement in Europe, and the U.S. Health and Retirement Study. Standardised estimates of national income inequality from 1960-2006 were taken from the Standardised World Income Inequality Database. We used multilevel regression methods to model the association between average 
inequality over this period and four measures of individual health: Objectively measured grip strength and lung function, and subjectively reported physical limitation and depressive symptoms.

Results We found that, after adjusting for individual and countrylevel covariates, exposure to higher average levels of inequality over the long-term was significantly negatively related to objectively measured grip strength and lung function, but unrelated to selfreported physical limitations or depressive symptoms.

Conclusion Our results show that long-term exposure to income inequality may indeed be detrimental to the physical health of older people. However, we found no evidence of an effect of inequality on subjectively reported limitations or depressive symptoms. This may be an effect of unmeasured covariates, or it may be due to the greater accuracy afforded by the objective health measures. To our knowledge this study represents the first direct evidence linking experience of inequality to the health of older people which has made use of either objective measures of health at the individual level, or a measure of inequality exposure over the long term.

\section{Public Health Interventions: Smoking}

\section{OP53 DEFINING THE LONG-TERM TREND IN A PUBLIC HEALTH INTERVENTION STUDY: A CAUTIONARY TALE}

doi:10.1136/jech-2012-201753.053

R Salway, A Gilmore, M Sims. Department for Health, University of Bath, Bath, UK

Background Numerous studies have reported on the impact of comprehensive smoke-free laws on population health. Many early studies have ignored the potential effect of the long-term trend of the health outcome, and when included, subsequent studies have focused on either linear or non-linear trends. However, the choice of appropriate trend is not always straightforward. We illustrate this by investigating the short-term impact of smoke-free legislation in England, introduced on 1st July 2007, on myocardial infarction mortality.

Methods We investigate the impact of the legislation using weekly counts of all cases aged 18 years or older residing in England with a primary cause of death of a myocardial infarction (ICD-10 I21) between July 2002 to December 2010 (providing 5 years pre-legislative and 3 years and 6 months post-legislative data). We compare a number of models based on an interrupted time series design with a quasi-Poisson generalised additive model that adjusts for seasonality and long-term trends.

Results Myocardial infarction mortality shows a marked decline over the study period. We identify two competing models. The first shows evidence of a complex interaction between the introduction of smoke-free legislation and the long-term trend. We observe an initial statistically significant reduction in mortality $(-8.5 \%, 95 \% \mathrm{CI}$ $-11.1 \%$ to $-5.8 \%$ ) coupled with a change in the long-term trend from a reduction of $4 \%$ over a six month period to a reduction of $3.5 \%$. The second model fits a nonlinear trend and shows no significant smoke-free effect. Both models offer an almost identical fit.

Conclusion Investigating small effects in the presence of a pronounced long-term trend is complicated by the limitations of the available data. In particular, it is not clear whether we observe a gradual change in the long term trend or a discrete effect directly attributable to the legislation. The two models have near-identical fitted values and GCV scores, but have very different interpretation. We conclude that the data alone are insufficient to distinguish between the two models and warn that overly-simplistic analyses in such situations may result in misleading conclusions.

\section{OP54 SHORT-TERM IMPACT OF THE SMOKEFREE LEGISLATION IN ENGLAND ON HOSPITAL ADMISSIONS FOR ASTHMA AMONG ADULTS}

doi:10.1136/jech-2012-201753.054

\section{Sims, A Gilmore. Department for Health, University of Bath, Bath, UK}

Background Comprehensive smokefree laws prohibiting smoking in enclosed public places and workplaces have now been introduced in several jurisdictions and there is a growing body of evidence documenting the immediate health benefits to adults, focusing primarily on hospital admissions for heart attacks. A few studies have examined the association between smokefree laws and asthma in adults, but these have limitations such as lacking appropriate adjustment for long-term trends, or having limited statistical power due to a small study population. In this study we investigated the short-term impact of the introduction of smokefree legislation in England on 1st July 2007 on hospital admissions for asthma in adults.

Methods The immediate effect of the legislation was investigated using monthly numbers of emergency admissions for asthma (primary diagnosis, ICD-10 code J45 and J46) in the nine Government Office Regions from April 1997 to December 2010, in the population aged 16 and over. The analysis was conducted using a quasi-Poisson generalised additive model that adjusted for seasonality and regionspecific, non-linear, long-term trends.

Results After adjusting for the long-term trend in admissions, we observed a $4.9 \%(95 \% \mathrm{CI}: 0.6,9.0)$ reduction in admissions for asthma immediately after introduction of smokefree legislation in the population as a whole. This implies that almost 1900 emergency admissions for asthma were prevented during the first year of the legislation. The reduction in admissions did not vary significantly across regions.

Conclusion Our finding, based on the largest study to date, adds to the expanding body of evidence that smokefree legislation is associated with positive health outcomes. Further research evaluating the impact of legislation on asthma admissions in other jurisdictions is needed in order to support these findings.

\section{OP55 SOCIOECONOMIC INEQUALITIES IN CHILDHOOD EXPOSURE TO SECONDHAND SMOKE BEFORE AND AFTER SMOKE- FREE LEGISLATION IN THREE UK COUNTRIES}

doi:10.1136/jech-2012-201753.055

${ }^{1}$ GF Moore, ${ }^{2 D}$ Currie, ${ }^{3} \mathrm{G}$ Gilmore, ${ }^{1} \mathrm{JC}$ Holliday, 'L Moore. 'DECIPHer, School of Social Sciences, Cardiff University, Cardiff, UK, UK; ${ }^{2} 2$ Child and Adolescent Health Research Unit, School of Medicine, University of St Andrews, St Andrews, UK, UK; ${ }^{3}$ Health Intelligence, Public Health Agency, Belfast, UK, UK

Background Secondhand smoke (SHS) exposure is higher among children from lower socioeconomic status (SES) families, contributing to the intergenerational reproduction of health inequalities. Legislation prohibiting smoking in enclosed public places was introduced in all UK countries between 2006 and 2007. Although opponents argued that it would displace smoking into the home, legislation has been associated with reduced childhood SHS exposure and increased prevalence of smoke-free homes. In some UK countries however, trends towards widening inequality in childhood SHS exposure have been reported following legislation. This paper combines datasets from 3 UK countries to examine socioeconomic patterning in childhood SHS exposure and smoking restrictions in homes and cars pre- and post-legislation.

Methods We conducted a repeat cross-sectional survey of 10,867 schoolchildren in 304 primary schools in Scotland, Wales and Northern Ireland. Children provided saliva for cotinine assay, completing questionnaires before and 12-months after legislation, including the Family Affluence Scale (a measure of socioeconomic status), and reports of smoking restrictions in homes and cars. Multinomial regression analyses assessed differences between survey years in SHS exposure and private smoking restrictions, with interaction terms to assess SES patterning in changes.

Results SHS exposure was highest, and private smoking restrictions least frequent, among lower SES children pre- and 